\title{
The Effect of Feeding Rosemary and Marjoram on Reproductive Performance of Rabbit Does Under Sinai Conditions
}

\author{
Badawi, Lobna A. M. A.; E. O. A. Bakr; M. R. M. Mousa and M. A. Abdel Ghaffar* \\ Department of Animal and Poultry Production, Faculty of Environmental Agricultural Sciences, Arish University
}

Received: $1 / 11 / .2016$

\begin{abstract}
This study was conducted to investigate the effects of dietary inclusion of different levels of Rosemary and Marjoram leaves on reproductive performance of rabbits. Forty eight New Zealand white (NZW) doe rabbits with initial body weight of $3.64 \pm 0.035 \mathrm{~kg}$ and aged 7 months were assigned to one of six dietary groups $(8 \mathrm{each})$. The $1^{\text {st }}$ group was of control group, the $2^{\text {nd }}$ and $3^{\text {rd }} \mathrm{d}$ groups were fed diets supplied with Rosemary dry leaves powder $(1.5 \%$ and $3.0 \%$, respectively), $4^{\text {th }}$ and $5^{\text {th }}$ groups were fed diets supplied with Marjoram dry leaves powder $(1.5 \%$ and $3.0 \%$, respectively), the $6^{\text {th }}$ group was fed the basal diet supplemented with $1.5 \%$ Rosemary dry leaves powder plus $1.5 \%$ Marjoram dry leaves powder. The body weight of does at mating, pre-natal weights, gestation length and kindling rate were insignificantly affected by Rosemary and Marjoram leaves inclusion in diets. Adding $1.5 \%$ Marjoram to the diets of rabbit does recorded significantly $(\mathrm{P} \leq 0.05)$ higher litter size and weight at 7, 14, 21 and 28 days of age (weaning age) than untreated does. Bunny weight at 7, 14, 21 and 28 days of age increased in rabbits fed $1.5 \%$ Rosemary. Rabbits feed supplemented with $1.5 \%$ Rosemary, $1.5 \%$ Marjoram and their mixture (1.5\% Rosemary and $1.5 \%$ Marjoram ) increased in total milk yield at birth -7 days, birth -14 days, birth -21 days and birth -28 days compared with the control rabbit does.
\end{abstract}

Keywords: Rabbit does, rosemary, marjoram, reproductive performance

\section{INTRODUCTION}

There is global awareness about the shortage of animal protein supply in the developing countries. The production of ruminants such as cattle, sheep and goats have not been able to bridge the gap because of their long production generation intervals, feed and fodder shortages, poor genetic make-up as for production traits and disease incidence are among other factors.

Nowadays, there have been rising recognition on the virtues of role that can be played by rabbit production in these developing countries as a means of relieving animal protein shortage crises. The advantages of rabbits are largely attributable to their high rate of reproduction and quick and precipitous maturity, rapid growth rate, high genetic selection potential, efficient use of feed and land utilization, limited competition on humans foods and high quality nutritious meat (Lukfahr and Cheeke, 1991).

There are a large number of feed additives available for inclusion in rabbit's diets to improve their performance (Ewuola et al., 2011). However, the use of chemical products especially (hormones and antibiotics), may cause unfavorable side effects. Moreover, there is evidence indicating that these products are currently considered as risky pollutants for human and may threaten their health on the long-run (Omer et al., 2013).

Many efforts have been devoted on using the green natural materials and/or medicinal plants as feed additives to improve the efficiency of feed utilization and productive performance (Aboul-fotouh et al., 1999) and these efforts are widely accepted. Several previous studies showed that adding medicinal plants and herbs to the diets of rabbits, chicks, sheep, cows or buffaloes improved their feed intake and nutrient digestibility (Mir et al., 1998; Aboul-fotouh et al., 1999 and ELAyek, 1999) feed conversion (Allam et al., 1999; Aboul-fotouh et al., 1999; Salem and El-Mahdy, 2001), body weight, body weight gain, growth performance and mortality rate (Ibrahim et al., 2004, Ibrahim, 2005 and Tipu et al., 2006), carcass traits (Evans and Pharm, 1975 ) and physical conditions of gut ecosystem (Guo, 2003)

Accordingly, the current study was carried out to evaluate the single and combined effects of Rosemary (Rosmarinus officinalis L.) and Marjoram (Origanum majorana L.) leaves on productive and reproductive performance of does rabbit under the prevailed circumstances of Sinai.

\section{MATERIALS AND METHODS}

The present study was carried out at rabbit research farm of Animal and Poultry Production Department, Faculty of Environmental Agriculture Science, Arish University. The geographical and climatic characteristics of this region (Long., 33.75E and Lat. $31.27 \mathrm{~N}$ ) is semi- arid with an average annual rain fall of about $94 \mathrm{~mm}$ and average ambient temperature of about $20.47^{\circ} \mathrm{C}$.

Rosemary (Rosmarinus officinalis $\quad$ L.) and Marjoram (Origanum majorana L.) plants were collected from a private commercial farm located in North Sinai governorate. The leaves were harvested, airdried under shade until the moisture of collected leaves reached $10 \%$. The dry leaves were finally milled, sieved $(1 \mathrm{~mm}$ mesh) and stored in a well tight polyethylene bags at room temperature $25^{\circ} \mathrm{C}$.

Forty eight New Zealand white (NZW) doe rabbits with initial body weight of $3.64 \pm 0.035 \mathrm{~kg}$ and aged 7 months during three parities from January to May were used in a complete randomized design experiment with six dietary treatment groups. The first group was control group fed a commercial diet without supplementation, second and third groups were fed the control diet supplied with Rosemary dry leaves powder $(1.5 \%$ and $3.0 \%$, respectively), the fourth and fifth groups were fed 
the control diet supplied with Marjoram dry leaves powder $(1.5 \%$ and $3.0 \%$, respectively), while the sixth group was fed the basal diet supplemented with $1.5 \%$ Rosemary dry leaves powder plus $1.5 \%$ Marjoram dry leaves powder.

Rabbits were fed commercial rabbit diet to cover their requirements according to NRC (1996). The formulation and chemical composition of commercial rabbit diet is shown in Table (1). Chemical analysis of diet was determined according to AOAC (2000).

Table (1): Ingredients of experimental diet used in this study

\begin{tabular}{|c|c|}
\hline Ingredients \% & Con. diet \\
\hline Yellow corn & 14 \\
\hline Barley grain & 10 \\
\hline Wheat bran & 27 \\
\hline Soybean meal $44 \%$ & 12 \\
\hline Alfalfa hay & 31.5 \\
\hline Molasses & 3 \\
\hline Dicalcium P. & 1.2 \\
\hline Calcium carbonate (lime stone) & 0.6 \\
\hline Sodium chloride (salt) & 0.3 \\
\hline Premix & 0.3 \\
\hline Methionine & 0.1 \\
\hline Total & 100 \\
\hline \multicolumn{2}{|l|}{$\begin{array}{l}\text { Calculated chemical } \\
\text { composition }\end{array}$} \\
\hline Crude protein (CP) & 17.38 \\
\hline Crude fiber (CF) & 12.27 \\
\hline Ether extract (EE) & 2.93 \\
\hline Digestible energy (Kcal/Kg) & 2769.70 \\
\hline
\end{tabular}

One kilogram of premix contain: vit. A $12000000 \mathrm{IU}$, vit.D $220000 \mathrm{IU}$, vit. E $1000 \mathrm{mg}$, vit. $\mathrm{K}_{3} 2000 \mathrm{mg}$, vit. $\mathrm{B}_{1} 1000$ $\mathrm{mg}$, vit. $\mathrm{B}_{2} 4000 \mathrm{mg}$, vit. $\mathrm{B}_{6} 100 \mathrm{mg}$, vit. $\mathrm{B}_{12} 10 \mathrm{mg}$, pantothenic acid $3.33 \mathrm{~g}$, biotin $33 \mathrm{mg}$, folic acid $0.83 \mathrm{~g}$, choline chloride 200 g, Zn 11.79 g, Mn 5 g, Fe 12.5 g, Cu $0.5 \mathrm{~g}$, I $33.3 \mathrm{mg}$, Se $16.6 \mathrm{mg}$ and $\mathrm{Mg} 66.7 \mathrm{~g}$.

Does were housed separately in individual wired cages $(60 \times 40 \times 35 \mathrm{~cm})$ and raised $100 \mathrm{~cm}$ from the concrete floor. Nest boxes $(30 \times 25 \times 30 \mathrm{~cm})$ were attached to the front sides of the cages five days prior to kindling and removed at 28 day of lactation (weaning age). All cages were equipped with feeders (made of galvanized steel sheets) and nipples (automatic drinkers). Feed and water were available ad-libitum during all experimental period.

Rabbits in all treatment groups were kept under similar managerial system and environmental conditions. Each doe was transferred to the cage of buck assigned for mating and returned back to her own cage after being mated and palpated for pregnancy 10 days after mating and those failed to conceive were returned back to the same mating buck to be remated within 12 hours, after kindling, litter size, litter weight and bunny weight were recorded. Bunny weight at birth, 7, 14, 21 and 28 (at weaning age) and daily gain weight up to weaning were also recorded. Body weight of does were recorded by weighing the does at the mating and prenatal. Kindling rate $\%=[$ No. of kindled does/No. of bunnies born (including dead bunnies)] x 100.Gestation period from fertile mating to kindling was recorded. Daily milk yield for each doe was measured individually twice/day (every 12 hours) by difference in weight of the pups after and before suckling. Averages of daily milk yield and total milk yield during the sucking period were recorded.

Data of doe rabbits were analyzed using general linear model procedure of SAS software (SAS Institute, 2004). Difference among treatment means were tested for significance using Duncan's Multiple Range Test (Duncan, 1955).

\section{RESULTS AND DISCUSSION}

\section{Approximate analysis of medicinal Plants:}

Data presented in Table (2) showed the chemical composition of Rosemary (Rosmarinus officinalis L.) and Marjoram (Origanum majorana L.) used in this study. The analysis was carried out to illustrate the proportional content of crude protein $(\mathrm{CP})$, ether extract (EE), crude fiber $(\mathrm{CF})$, ash and nitrogen free extract (NEE) in this plants. The highest value of crude protein, crude fiber and ash were observed of Marjoram (12.27, 15.03 and $14.22 \%$ ) while the lowest $(5.91,14.23$ and $5.26 \%$ ) were recorded of Rosemary. However, the highest value of ether extract and nitrogen free extract were recorded of Rosemary (6.83 and 67.77\%) while, the lowest value $(2.27$ and $56.21 \%)$ were recorded of Marjoram.

Results showed that the value of CP in Rosemary was similar to those reported by Radwan (2003), Ghazalah and Ali (2008), Mohamed (2009) and Osman et al. (2010), but it was lower than this reported by Othman (2005). Values of EE, NFE and ash were higher than those reported by (Radwan, 2003; Ghazalah and Ali, 2008; Othman, 2005; Mohamed, 2009; Osman et al., 2010).

In addition, value of CP in Marjoram was higher than those reported by Osman et al. (2010) and Ali (2014), but it was lower than those reported by Abaza (2001), Abdo et al. (2003) and Ahmed and AbdelGhany (2015). However, Ash value was lower than those values reported by Abdo et al. (2003) and Ahmed and Abdel-Ghany (2015), but it was higher than this reported by Abaza (2001), Osman et al. (2010) and Ali (2014). Moreover, value of EE and NFE was higher than those reported by (Radwan, 2003; Ghazalah and Ali, 2008; Othman, 2005; Mohamed 2009; Osman et al., 2010). The chemical constituents of tested feed additives may vary according to processing of these plants, climatic conditions under which the seeds were grown and the kind of soil (Osman et al., 2010). 
Table (2):.Chemical analysis (\%) of medicinal plants and the experimental diets

\begin{tabular}{|c|c|c|c|c|c|c|c|}
\hline \multirow{2}{*}{ Items } & \multirow{2}{*}{ DM } & \multicolumn{6}{|c|}{$\%$ On DM basis } \\
\hline & & OM & $\mathbf{C P}$ & $\mathbf{E E}$ & $\mathbf{C F}$ & NFE & ASH \\
\hline Rosemary & 89.96 & 94.77 & 5.02 & 9.23 & 21.71 & 58.81 & 5.23 \\
\hline Marjoram & 89.82 & 91.38 & 13.22 & 3.21 & 19.07 & 55.88 & 8.62 \\
\hline \multicolumn{8}{|c|}{ Chemical composition of experimental diets given to NZW rabbits } \\
\hline Control & 90.53 & 91.40 & 17.38 & 2.93 & 12.26 & 58.83 & 8.60 \\
\hline Rosemary $1.5 \%$ & 90.20 & 91.45 & 17.19 & 3.02 & 12.40 & 58.84 & 8.55 \\
\hline Rosemary 3\% & 89.58 & 91.50 & 17.01 & 3.20 & 12.54 & 58.75 & 8.50 \\
\hline Marjoram 1.5\% & 90.20 & 91.40 & 17.32 & 2.93 & 12.36 & 58.79 & 8.60 \\
\hline Marjoram 3\% & 90.50 & 91.40 & 17.26 & 2.94 & 12.46 & 58.74 & 8.60 \\
\hline Mixture & 90.40 & 91.45 & 17.13 & 3.03 & 12.50 & 58.79 & 8.55 \\
\hline
\end{tabular}

Body weight and kindling rate of doe rabbits:

Feeding diet supplemented with medicinal plants did not affect body weight of does at mating, pre- natal weights and gestation length $(\mathrm{P}>0.05)$ compared with the control group (Table 3 ). In contrary, kindling rate was increased in dose fed diet supplemented with Rosemary, Marjoram and their mixture groups (Table
9). The present results are in agreement with those obtained by Seleem et al. (2007) who found that adding $3 \%$ Marjoram (Origanum majorana) to the diets of rabbit does significantly $(\mathrm{P} \leq 0.05)$ increased kindling rate compared with untreated does. They found kindling rates for treated and untreated does to be 80.92 and $60.53 \%$, respectively.

Table (3): Effect of dietary levels of natural feed additives on body weight at mating and pre-natal weights, gestation length and Kindling rate of New Zealand white rabbits.

\begin{tabular}{|c|c|c|c|c|c|c|}
\hline \multirow{3}{*}{ Items } & \multicolumn{6}{|c|}{ Experimental diets } \\
\hline & \multirow{2}{*}{ Control } & \multicolumn{2}{|c|}{ Rosemary } & \multicolumn{2}{|c|}{ Marjoram } & \multirow[t]{2}{*}{ Mixture } \\
\hline & & $1.5 \%$ & $3 \%$ & $1.5 \%$ & $3 \%$ & \\
\hline Mating weight (kg) & $\begin{array}{c}3.27 \\
\pm 0.066\end{array}$ & $\begin{array}{c}3.23 \\
\pm 0.067\end{array}$ & $\begin{array}{c}3.19 \\
\pm 0.077\end{array}$ & $\begin{array}{c}3.32 \\
\pm 0.063\end{array}$ & $\begin{array}{c}3.31 \\
\pm 0.076\end{array}$ & $\begin{array}{c}3.32 \\
\pm 0.059\end{array}$ \\
\hline Pre-natal weight (kg) & $\begin{array}{c}3.44 \\
\pm 0.055\end{array}$ & $\begin{array}{c}3.55 \\
\pm 0.056\end{array}$ & $\begin{array}{c}3.60 \\
\pm 0.061\end{array}$ & $\begin{array}{c}3.45 \\
\pm 0.063\end{array}$ & $\begin{array}{c}3.34 \\
\pm 0.065\end{array}$ & $\begin{array}{c}3.22 \\
\pm 0.057\end{array}$ \\
\hline Gestation length (day) & $\begin{array}{c}30.40 \\
\pm 0.026\end{array}$ & $\begin{array}{c}30.50 \\
\pm 0.025\end{array}$ & $\begin{array}{c}31.00 \\
\pm 0.022\end{array}$ & $\begin{array}{c}30.00 \\
\pm 0.030\end{array}$ & $\begin{array}{c}30.10 \\
\pm 0.027\end{array}$ & $\begin{array}{c}30.60 \\
\pm 0.028\end{array}$ \\
\hline Kindling rate (\%) & $\begin{array}{c}77.50 \\
\pm 0.014\end{array}$ & $\begin{array}{c}83.30 \\
\pm 0.020\end{array}$ & $\begin{array}{c}87.0 \\
\pm 0.018\end{array}$ & $\begin{array}{c}85.90 \\
\pm 0.023\end{array}$ & $\begin{array}{c}80.10 \\
\pm 0.018\end{array}$ & $\begin{array}{c}87.2 \\
\pm 0.033\end{array}$ \\
\hline
\end{tabular}

\section{Litter size and weight:}

Data presented in Table (4) showed the effect of medicinal plants on litter size at birth, 7, 14, 21 and 28 days (weaning). Results clearly indicated that litter size at birth was not significantly influenced by medicinal plants supplemented to doe diets. Rabbits fed diet supplemented with $1.5 \%$ Marjoram achieved the highest $(\mathrm{P} \leq 0.05)$ litter size at $7,14,21$ and 28 days
(6.05, 6.00, 6.00 and 6.00 , respectively) compared with control group $(5.92,5.83,5.83$ and 5.75 , respectively). However, rabbits fed diets supplemented with $3 \%$ Rosemary recorded the lowest $(4.43,4.43,4.43$ and 4.50) litter size at 7, 14, 21 and 28 days, respectively. These results are in agreement with Seleem et al. (2007) who found that adding 3\% Origanum majorana (Marjoram) to the diets of rabbit does significantly 
increased litter size at birth (7.3) compared with the untreated does (5.1). Also, Shehata et al. (2007) who reported that the number of a live kids/doe at birth were higher in (Matricaria chamomila) flower treated groups (2.11 \& 2.23) compared with 1.97 for control group. In addition, El-Hammady and Abdel-Kareem (2015) observed an improvement in litter size at birth and at weaning of rabbits fed diet supplemented with $1.0 \mathrm{~g}$ herbal dried seeds, consisting of 50\% fenugreek; $30 \%$ caraway and $10 \%$ of both fennel and dill than the control group.

Concerning litter weight (Table 4) demonstrated that the litter weight at birth, 7, 14, $21 \mathrm{~d}$ and 28 days (weaning) significantly $(\mathrm{P} \leq 0.05)$ increased (409.45, $834.65,1361.30,2021.00$ and $2386.50 \mathrm{~g}$, respectively) in group fed diet supplemented with 3\% Marjoram compared to control $(356.75,648.92,1028.25,1537.70$ and $1874.60 \mathrm{~g}$, respectively). However, Rabbits fed diets supplemented with 3\% Rosemary recorded the lowest litter weight $(336.07,573.86,945.93,1439.00$ and $1831.70 \mathrm{~g}$, respectively) at birth, 7, 14, 21 and 28 days, respectively. This result agreed with Seleem et al. (2007) who found that adding 3\% Origanum majorana (Marjoram) to the diets of rabbit does significantly improved litter weight Moreover, Shehata et al. (2007) found that feeding (Matricaria chamomila) flower in Zaraibi does ration had positive effect on new born kids weight. In addition, Gaafar et al. (2014) indicated that doe rabbits fed diets supplemented with a combination of pumpkin and black seeds oils (2.5 g PS oil plus $2.5 \mathrm{~g}$ $\mathrm{BS}$ oil $/ \mathrm{kg}$ diet) showed the better litter weight at birth, 7, 14, 21 and 28 days than those in control group.

Table (4): Effect of dietary levels of natural feed additives on litter size and weight and mortality of New Zealand white rabbits

\section{Experimental diets}

\begin{tabular}{|c|c|c|c|c|c|c|c|}
\hline \multirow[t]{2}{*}{ Items } & \multirow[t]{2}{*}{ at } & \multirow{2}{*}{ Control } & \multicolumn{2}{|c|}{ Rosemary } & \multicolumn{2}{|c|}{ Marjoram } & \multirow{2}{*}{ Mixture } \\
\hline & & & $1.5 \%$ & $3 \%$ & $1.5 \%$ & $3 \%$ & \\
\hline \multirow{5}{*}{ Litter size } & Birth & $\begin{array}{c}6.17 \\
\pm 0.34\end{array}$ & $\begin{array}{c}6.00 \\
\pm 0.41\end{array}$ & $\begin{array}{c}6.00 \\
\pm 0.41\end{array}$ & $\begin{array}{c}6.45 \\
\pm 0.27\end{array}$ & $\begin{array}{c}6.21 \\
\pm 0.32\end{array}$ & $\begin{array}{l}5.94 \\
\pm 0.2\end{array}$ \\
\hline & 7 days & $\begin{array}{c}5.92^{\mathrm{a}} \\
\pm 0.36\end{array}$ & $\begin{array}{l}5.38^{\mathrm{ab}} \\
\pm 0.37\end{array}$ & $\begin{array}{l}4.43^{\mathrm{ab}} \\
\pm 0.29\end{array}$ & $\begin{array}{c}6.05^{\mathrm{a}} \\
\pm 0.28\end{array}$ & $\begin{array}{l}5.07^{\mathrm{ab}} \\
\pm 0.49\end{array}$ & $\begin{array}{l}5.44^{\mathrm{ab}} \\
\pm 0.26\end{array}$ \\
\hline & 14 days & $\begin{array}{l}5.83^{\mathrm{a}} \\
\pm 0.3\end{array}$ & $\begin{array}{l}5.33^{\mathrm{ab}} \\
\pm 0.36\end{array}$ & $\begin{array}{c}4.43^{\mathrm{b}} \\
\pm 0.29\end{array}$ & $\begin{array}{c}6.00^{\mathrm{a}} \\
\pm 0.31\end{array}$ & $\begin{array}{l}4.93^{\mathrm{ab}} \\
\pm 0.51\end{array}$ & $\begin{array}{l}5.70^{\mathrm{ab}} \\
\pm 0.20\end{array}$ \\
\hline & 21 days & $\begin{array}{l}5.83^{\mathrm{ab}} \\
\pm 0.39\end{array}$ & $\begin{array}{l}5.33^{\mathrm{abc}} \\
\pm 0.36\end{array}$ & $\begin{array}{c}4.43^{\mathrm{c}} \\
\pm 0.29\end{array}$ & $\begin{array}{c}6.00^{\mathrm{a}} \\
\pm 0.29\end{array}$ & $\begin{array}{l}6.00^{\mathrm{bc}} \\
\pm 0.49\end{array}$ & $\begin{array}{l}5.11^{\mathrm{abc}} \\
\pm 0.18\end{array}$ \\
\hline & Weaning (28d) & $\begin{array}{l}5.75^{\mathrm{ab}} \\
\pm 0.35\end{array}$ & $\begin{array}{l}5.48^{\mathrm{abc}} \\
\pm 0.36\end{array}$ & $\begin{array}{c}4.50^{c} \\
\pm 0.33\end{array}$ & $\begin{array}{c}6.00^{\mathrm{a}} \\
\pm 0.29\end{array}$ & $\begin{array}{l}4.85^{\mathrm{bc}} \\
\pm 0.48\end{array}$ & $\begin{array}{l}5.11^{\mathrm{abc}} \\
\pm 0.18\end{array}$ \\
\hline \multirow{5}{*}{$\begin{array}{c}\text { litter } \\
\text { weight } \\
\text { (gm.) }\end{array}$} & Birth (a total) & $\begin{array}{l}356.75^{\mathrm{ab}} \\
\pm 27.56\end{array}$ & $\begin{array}{l}376.29^{\mathrm{ab}} \\
\pm 24.84\end{array}$ & $\begin{array}{l}336.07^{b} \\
\pm 20.89\end{array}$ & $\begin{array}{l}409.45^{\mathrm{a}} \\
\pm 14.83\end{array}$ & $\begin{array}{l}370.86^{\mathrm{ab}} \\
\pm 23.51\end{array}$ & $\begin{array}{l}386.39^{\mathrm{ab}} \\
\pm 17.00\end{array}$ \\
\hline & 7 days & $\begin{array}{c}648.92^{\mathrm{bcd}} \\
\pm 30.11\end{array}$ & $\begin{array}{l}748.14^{\mathrm{ab}} \\
\pm 43.15\end{array}$ & $\begin{array}{l}573.86^{\mathrm{d}} \\
\pm 38.65\end{array}$ & $\begin{array}{l}834.65^{\mathrm{a}} \\
\pm 24.40\end{array}$ & $\begin{array}{l}610.36^{\mathrm{cd}} \\
\pm 51.50\end{array}$ & $\begin{array}{l}692.78^{b c} \\
\pm 24.66\end{array}$ \\
\hline & 14 days & $\begin{array}{c}1028.25^{\mathrm{c}} \\
\pm 46.30\end{array}$ & $\begin{array}{c}1236.29^{\mathrm{ab}} \\
\pm 70.73\end{array}$ & $\begin{array}{l}945.93^{c} \\
\pm 71.59\end{array}$ & $\begin{array}{c}1361.30^{\mathrm{a}} \\
\pm 45.89\end{array}$ & $\begin{array}{l}959.14^{\mathrm{c}} \\
\pm 83.50\end{array}$ & $\begin{array}{c}1107.39^{\mathrm{bc}} \\
\pm 42.97\end{array}$ \\
\hline & 21 days & $\begin{array}{c}1537.70^{\mathrm{c}} \\
\pm 59.04\end{array}$ & $\begin{array}{l}1873.70^{\mathrm{ab}} \\
\pm 102.41\end{array}$ & $\begin{array}{l}1439.00^{\mathrm{c}} \\
\pm 122.02\end{array}$ & $\begin{array}{c}2021.00^{\mathrm{a}} \\
\pm 71.70\end{array}$ & $\begin{array}{c}1430.40^{\mathrm{c}} \\
\pm 117.1\end{array}$ & $\begin{array}{c}1642.9^{\mathrm{bc}} \\
\pm 73.05\end{array}$ \\
\hline & Weaning (28) & $\begin{array}{c}1874.60^{\mathrm{b}} \\
\pm 69.83\end{array}$ & $\begin{array}{l}2320.20^{\mathrm{a}} \\
\pm 142.19\end{array}$ & $\begin{array}{l}1831.70^{\mathrm{b}} \\
\pm 167.15\end{array}$ & $\begin{array}{c}2386.50^{\mathrm{a}} \\
\pm 98.58\end{array}$ & $\begin{array}{l}1765.10^{\mathrm{b}} \\
\pm 144.86\end{array}$ & $\begin{array}{c}2013.80^{\mathrm{ab}} \\
\pm 106.77\end{array}$ \\
\hline \multirow{4}{*}{$\begin{array}{l}\text { Mortality } \\
\text { rate }\end{array}$} & Birth -7 days & $\begin{array}{l}03.10^{\mathrm{b}} \\
\pm 0.02\end{array}$ & $\begin{array}{c}6.20^{\mathrm{b}} \\
\pm 0.02\end{array}$ & $\begin{array}{c}19.30^{\mathrm{a}} \\
\pm 0.063\end{array}$ & $\begin{array}{c}8.11^{b} \\
\pm 0.03\end{array}$ & $\begin{array}{l}24.00^{\mathrm{a}} \\
\pm 0.0^{4}\end{array}$ & $\begin{array}{c}8.12^{\mathrm{b}} \\
\pm 0.03\end{array}$ \\
\hline & Birth -14 days & $\begin{array}{l}5.60^{\mathrm{c}} \\
\pm 0.02\end{array}$ & $\begin{array}{l}7.00^{\mathrm{c}} \\
\pm 0.02\end{array}$ & $\begin{array}{c}21.00^{\mathrm{ab}} \\
\pm 0.07\end{array}$ & $\begin{array}{l}9.00^{\mathrm{c}} \\
\pm 0.07\end{array}$ & $\begin{array}{l}24.00^{\mathrm{a}} \\
\pm 0.04\end{array}$ & $\begin{array}{l}12.00^{\mathrm{bc}} \\
\pm 0.03\end{array}$ \\
\hline & Birth -21 days & $\begin{array}{l}6.00^{\mathrm{c}} \\
\pm 0.02\end{array}$ & $\begin{array}{l}7.00^{\mathrm{c}} \\
\pm 0.02\end{array}$ & $\begin{array}{c}22.00^{\mathrm{ab}} \\
\pm 0.07\end{array}$ & $\begin{array}{l}9.00^{\mathrm{c}} \\
\pm 0.03\end{array}$ & $\begin{array}{l}24.00^{\mathrm{a}} \\
\pm 0.04\end{array}$ & $\begin{array}{l}13.00^{\mathrm{bc}} \\
\pm 0.03\end{array}$ \\
\hline & Birth -28 days & $\begin{array}{c}7.00^{\mathrm{b}} \\
\pm 0.02\end{array}$ & $\begin{array}{c}7.00^{\mathrm{b}} \\
\pm 0.02\end{array}$ & $\begin{array}{l}22.00^{\mathrm{a}} \\
\pm 0.07\end{array}$ & $\begin{array}{c}7.00^{\mathrm{b}} \\
\pm 0.04\end{array}$ & $\begin{array}{l}24.00^{\mathrm{a}} \\
\pm 0.05\end{array}$ & $\begin{array}{l}13.00^{b c} \\
\pm 0.03\end{array}$ \\
\hline
\end{tabular}


Concerning mortality rate results in Table (4) indicated that increasing the Rosemary or Marjoram up to $3 \%$ in rabbits diets significantly $(\mathrm{P}<0.05)$ increased the mortality rate compared with the control group and other treatments. In contrary El-Khilany et al. (2009) indicated that feeding laying hens on diets supplemented with karkade (Hibiscus sabdariffa) seed decreased $(\mathrm{P} \leq 0.05)$ mortality rate. However, Mukhtar (2009) showed that feeding karkade seed to broiler chicks showed no effect on the mortality rate of chicks. In addition, Ocak et al. (2008) found that feeding diets supplemented with thyme (Thymus vulgaris) leaves, mortality was lower in birds fed the thyme diets than in birds fed control diets for the entire growing period in broilers. The increase of rabbit pre-weaning mortality was associated with an increase in litter size at birth and reduction of remating interval period, although doe milk yield appeared to be the most important factor in this respect. Thus, all factors which may decrease doe rabbit milk (nutrition, management of the rabbitry, climatic conditions and doe diseases), increase preweaning mortality (Rashwan and Marai, 2000).

Mean bunny body weight gain:

Feeding the doe rabbits on Rosemary, Marjoram and their mixture supplementation increased $(\mathrm{P} \leq 0.05)$ mean bunny weight at 7, 14, 21 and 28 days (weaning) except at birth compared with control group (Table, 5). Rabbits fed diets supplemented with 1.5\% Rosemary had the highest mean bunny weight (143.54, 238.37, 363.08 and 431.14) at 7, 14, 21 and 28 days, respectively. However, the control group recorded the lowest mean bunny weight values (111.68, 181.14, 271.48 and $333.51 \mathrm{~g})$, respectively.

Feeding doe rabbits on diets supplemented with $1.5 \%$ Rosemary significantly $(\mathrm{P} \leq 0.05)$ increased bunny weight gain than control group (79.33, 174.16, 298.86 and $366.93 \mathrm{~g} /$ day) during the periods from birth -7 days, birth -14 days, birth -21 days and birth -28 days respectively. However, rabbits fed control diet without any supplemented achieved the lowest bunny weight gain $(53.20,122.69,213.03$ and $275.06 \mathrm{~g} /$ day) as presented in Table (5). These results agreed with Elkholy et al. (2012) who found that the doses of Black Baladi rabbit supplemented with $1000 \mathrm{~g}$ cinnamon (Cinnamomum zeylanicum) powder had significantly $(\mathrm{P} \leq 0.05)$ higher bunny weight at birth and at weaning than the control group. The improvement in bunny weight gain may be attributed to increased feed intake and feed conversion and, milk yield, and to enhancement in the metabolism of essential and volatile oils included in medicinal plants (Evans and Pharm, 1975).

Table (5): Effect of dietary levels of natural feed additives on mean bunny weight gain of New Zealand White rabbits

\begin{tabular}{|c|c|c|c|c|c|c|c|}
\hline \multirow{3}{*}{ Items } & \multirow{3}{*}{ at } & \multicolumn{6}{|c|}{ Experimental diets } \\
\hline & & \multirow[t]{2}{*}{ Control } & \multicolumn{2}{|c|}{ Rosemary } & \multicolumn{2}{|c|}{ Marjoram } & \multirow[t]{2}{*}{ Mixture } \\
\hline & & & $1.5 \%$ & $3 \%$ & $1.5 \%$ & $3 \%$ & \\
\hline \multirow{5}{*}{$\begin{array}{l}\text { Mean bunny } \\
\text { weight (gm.) }\end{array}$} & Birth (a total) & $\begin{array}{l}58.45 \\
\pm 4.27\end{array}$ & $\begin{array}{l}64.21 \\
\pm 2.47\end{array}$ & $\begin{array}{l}58.47 \\
\pm 4.36\end{array}$ & $\begin{array}{l}64.42 \\
\pm 2.17\end{array}$ & $\begin{array}{l}60.39 \\
\pm 3.41\end{array}$ & $\begin{array}{l}65.15 \\
\pm 2.18\end{array}$ \\
\hline & 7 days & $\begin{array}{c}111.68^{\mathrm{c}} \\
\pm 5.22\end{array}$ & $\begin{array}{c}143.54^{\mathrm{a}} \\
\pm 5.40\end{array}$ & $\begin{array}{c}130.72^{\mathrm{ab}} \\
\pm 4.22\end{array}$ & $\begin{array}{c}141.44^{\mathrm{ab}} \\
\pm 5.41\end{array}$ & $\begin{array}{c}125.26^{\mathrm{bc}} \\
\pm 6.47\end{array}$ & $\begin{array}{c}129.30^{\mathrm{ab}} \\
\pm 4.33\end{array}$ \\
\hline & 14 days & $\begin{array}{c}181.14^{\mathrm{c}} \\
\pm 9.41\end{array}$ & $\begin{array}{c}238.37^{\mathrm{a}} \\
\pm 7.90\end{array}$ & $\begin{array}{c}214.56^{\mathrm{ab}} \\
\pm 8.61\end{array}$ & $\begin{array}{c}232.82^{\mathrm{a}} \\
\pm 9.86\end{array}$ & $\begin{array}{c}200.99^{\mathrm{bc}} \\
\pm 7.75\end{array}$ & $\begin{array}{c}215.66^{\mathrm{ab}} \\
\pm 6.07\end{array}$ \\
\hline & 21 days & $\begin{array}{l}271.48^{\mathrm{c}} \\
\pm 13.31\end{array}$ & $\begin{array}{l}363.08^{\mathrm{a}} \\
\pm 12.79\end{array}$ & $\begin{array}{c}324.18^{\mathrm{ab}} \\
\pm 14.76\end{array}$ & $\begin{array}{c}346.56^{\mathrm{ab}} \\
\pm 16.05\end{array}$ & $\begin{array}{c}306.27^{\mathrm{bc}} \\
\pm 13.06\end{array}$ & $\begin{array}{c}321.98^{\mathrm{ab}} \\
\pm 9.76\end{array}$ \\
\hline & $\begin{array}{c}\text { Weaning } \\
\text { (28d) }\end{array}$ & $\begin{array}{l}333.51^{\mathrm{c}} \\
\pm 13.78\end{array}$ & $\begin{array}{l}431.14^{\mathrm{a}} \\
\pm 12.22\end{array}$ & $\begin{array}{c}404.78^{\mathrm{ab}} \\
\pm 18.87\end{array}$ & $\begin{array}{c}406.88^{\mathrm{ab}} \\
\pm 18.33\end{array}$ & $\begin{array}{l}374.82^{\mathrm{c}} \\
\pm 14.65\end{array}$ & $\begin{array}{c}393.60^{\mathrm{ab}} \\
\pm 15.98\end{array}$ \\
\hline \multirow{4}{*}{$\begin{array}{l}\text { Bunny weight } \\
\text { gain (gm.) }\end{array}$} & Birth -7 days & $\begin{array}{l}53.20^{\mathrm{b}} \\
\pm 4.12\end{array}$ & $\begin{array}{l}79.33^{\mathrm{a}} \\
\pm 4.73\end{array}$ & $\begin{array}{l}72.2^{\mathrm{a}} \\
\pm 4.00\end{array}$ & $\begin{array}{l}77.0^{\mathrm{a}} \\
\pm 5.03\end{array}$ & $\begin{array}{c}64.87^{\mathrm{ab}} \\
\pm 6.11\end{array}$ & $\begin{array}{c}64.15^{\mathrm{ab}} \\
\pm 4.76\end{array}$ \\
\hline & Birth -14 days & $\begin{array}{c}122.69^{c} \\
\pm 9.15\end{array}$ & $\begin{array}{c}174.16^{\mathrm{a}} \\
\pm 7.73\end{array}$ & $\begin{array}{c}156.09^{\mathrm{ab}} \\
\pm 8.47\end{array}$ & $\begin{array}{c}168.40^{\mathrm{a}} \\
\pm 9.70\end{array}$ & $\begin{array}{c}140.60^{\mathrm{bc}} \\
\pm 8.32\end{array}$ & $\begin{array}{c}150.51^{\mathrm{ab}} \\
\pm 6.38\end{array}$ \\
\hline & Birth -21 days & $\begin{array}{l}213.03^{c} \\
\pm 13.79\end{array}$ & $\begin{array}{l}298.86^{\mathrm{a}} \\
\pm 12.46\end{array}$ & $\begin{array}{c}265.71^{\mathrm{ab}} \\
\pm 14.83\end{array}$ & $\begin{array}{c}282.14^{\mathrm{ab}} \\
\pm 15.73\end{array}$ & $\begin{array}{c}245.87^{\mathrm{bc}} \\
\pm 14.06\end{array}$ & $\begin{array}{c}256.82^{\mathrm{ab}} \\
\pm 10.18\end{array}$ \\
\hline & Birth -28 days & $\begin{array}{l}275.06^{\mathrm{c}} \\
\pm 14.81\end{array}$ & $\begin{array}{l}366.93^{\mathrm{a}} \\
\pm 11.76\end{array}$ & $\begin{array}{c}346.30^{\mathrm{ab}} \\
\pm 20.34\end{array}$ & $\begin{array}{c}342.46^{\mathrm{ab}} \\
\pm 17.88\end{array}$ & $\begin{array}{c}314.42^{\mathrm{bc}} \\
\pm 15.69\end{array}$ & $\begin{array}{c}328.44^{\mathrm{ab}} \\
\pm 16.12\end{array}$ \\
\hline
\end{tabular}




\section{Milk yield traits:}

Data in Table (6) showed that there were significant $(\mathrm{P}<0.05)$ differences in milk production for doe rabbits fed diets supplemented with medicinal plants during suckling period.

Does rabbits fed 1.5\% Rosemary, 1.5\% Marjoram and mixture $1.5 \%$ Rosemary $+1.5 \%$ Marjoram had the highest $(\mathrm{P} \leq 0.05)$ values of total milk yield during the birth -7 days, birth -14 days, birth -21 days and birth -28 days compared with the control rabbit does. The best values for the period from birth to 28 were recorded with 1.5\% Marjoram (5405.70 g) followed with 1.5\% Rosemary (5370.33 g) the lowest $(3646.08 \mathrm{~g})$ one recorded in the control group. The present results are in agreement with Seleem et al. (2007) who found that adding 3\% Marjoram (Origanum majorana) to the diet of rabbit does significantly increased milk yield/ doe compared with untreated does. In addition, Chiofalo et al. (2012) found that Rosemary (Rosmarinus officinalis) extract supplementation to dairy ewes affected $(\mathrm{P}<0.05)$ milk yield, and quantitative production of protein and casein, lactose and fat in their milk.

The improvements of Rosemary on milk yield may be attributed to "bio-functional compounds" which positively affected milk and alleviated the stress associated with lactation in animals (Chiofalo et al., 2012). Medicinal plants possess a lactogenic activity with a favorable enhancement ability in increasing serum prolactin level which is the principal lactogenic hormone secreted by anterior pituitary (Okasha et al., 2008; Gaya et al., 2009).

Table (6): Effect of dietary levels of natural feed additives on milk yield and milk conversion of New Zealand White rabbits

Treatments

\begin{tabular}{|c|c|c|c|c|c|c|c|}
\hline \multirow{3}{*}{ Items } & \multirow{3}{*}{ at } & & & & & & \\
\hline & & \multirow[t]{2}{*}{ Control } & \multicolumn{2}{|c|}{ Rosemary } & \multicolumn{2}{|c|}{ Marjoram } & \multirow[t]{2}{*}{ Mixture } \\
\hline & & & $1.5 \%$ & $3 \%$ & $1.5 \%$ & $3 \%$ & \\
\hline \multirow{4}{*}{$\begin{array}{l}\text { Milk yield } \\
\text { (gm.) }\end{array}$} & Birth -7 days & $\begin{array}{l}451.50^{b} \\
\pm 19.81\end{array}$ & $\begin{array}{l}761.00^{\mathrm{a}} \\
\pm 42.01\end{array}$ & $\begin{array}{l}489.50^{b} \\
\pm 50.66\end{array}$ & $\begin{array}{l}870.80^{\mathrm{a}} \\
\pm 48.78\end{array}$ & $\begin{array}{l}584.50^{b} \\
\pm 42.79\end{array}$ & $\begin{array}{l}733.44^{\mathrm{a}} \\
\pm 48.31\end{array}$ \\
\hline & Birth -14 days & $\begin{array}{c}1141.58^{\mathrm{c}} \\
\pm 65.85\end{array}$ & $\begin{array}{l}1970.00^{\mathrm{a}} \\
\pm 100.71\end{array}$ & $\begin{array}{l}1236.50^{\mathrm{bc}} \\
\pm 106.27\end{array}$ & $\begin{array}{c}2196.60^{\mathrm{a}} \\
\pm 92.31\end{array}$ & $\begin{array}{l}1463.50^{\mathrm{b}} \\
\pm 117.79\end{array}$ & $\begin{array}{c}1903.61^{\mathrm{a}} \\
\pm 84.91\end{array}$ \\
\hline & Birth -21 days & $\begin{array}{l}2511.83^{d} \\
\pm 133.02\end{array}$ & $\begin{array}{c}4347.00^{\mathrm{ab}} \\
\pm 170.82\end{array}$ & $\begin{array}{c}2617.00^{\mathrm{dc}} \\
\pm 159.45\end{array}$ & $\begin{array}{l}4370.50^{\mathrm{a}} \\
\pm 191.96\end{array}$ & $\begin{array}{l}3143.50^{c} \\
\pm 193.85\end{array}$ & $\begin{array}{l}4154.89^{b} \\
\pm 141.56\end{array}$ \\
\hline & Birth -28 days & $\begin{array}{l}3646.08^{c} \\
\pm 133.02\end{array}$ & $\begin{array}{l}5370.33^{\mathrm{a}} \\
\pm 208.05\end{array}$ & $\begin{array}{c}4542.00^{\mathrm{ab}} \\
\pm 203.73\end{array}$ & $\begin{array}{l}5405.70^{\mathrm{a}} \\
\pm 204.73\end{array}$ & $\begin{array}{c}4103.50^{\mathrm{ab}} \\
\pm 207.95\end{array}$ & $\begin{array}{l}5229.78^{\mathrm{a}} \\
\pm 219.76\end{array}$ \\
\hline
\end{tabular}

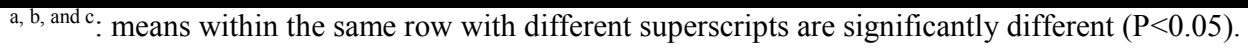

\section{CONCLUSION}

Based on the foregoing results, it could be concluded that diets supplemented with $1.5 \%$ Rosemary or Marjoram leaves could be used efficiency in feeding New Zealand white does without adverse, under North Sinai conditions.

\section{REFERENCES}

Abaza, I. M. (2001). The use of some medicinal plants additives in broiler diets. Ph.D. Thesis, Faculty of Agriculture, Alexandria University, Egypt.

Abdo, Zeinab M. A., A. Z. M. Soliman and S. Olfat Barakat (2003). Effect of hot Pepper and Marjoram as feed additives on the growth performance and the microbial population of the gastrointestinal tract of broilers. Egypt. Poult. Sci., 23 (I): 91-113.

Aboul-fotouh, G. E., S. M. Allam, E. Shehat and S. N. Abdel-Azeem (1999). Effect of some medicinal plants as feed additives on performance of growing sheep. Egypt J. Nutr. and Feeds, 2: 79-87.

Ahmed, E. G. and A. M. Abdel-Ghany (2015) The effect of Origanum majorana supplementation on growth performance blood parameters and meat quality in BUT9 commercial turkeys. Journal of Animal, Poultry and Fish Production. Suez Canal University, (3): 17 -29.

Ali, A. H. H. (2014). Productive performance and immune response of broiler chicks as affected by dietary Marjoram leaves powder. Egypt. Poult. Sci., 34(I): 57-70.

Allam, S. M., M. EL-Hosseiny, A. M. Abdel-Gawad, S. A. EL-Saadany and A. M. Zeid (1999). Medicinal herbs and plants as feed additives for ruminants. 1- Effect of using some medicinal herbs and plants as feed additives on Zaraibi goat performance. Egypt J. Nutr. and Feeds, 2: 349-365. 
A. O. A. C. (2000). Official Methods of Analysis. Chemists. $17^{\text {th }}$ Ed. Association of Official Analytical Chemists. Washington, D.C. USA.

Chiofalo, V., L. Liotta, R. Fiumanò, E. R. Benedetta and B. Chiofalo (2012). Influence of dietary supplementation of Rosmarinus officinalis L. on performances of dairy ewes organically managed. Small Ruminant Research, 104: 122-128.

Duncan, D. B. (1955). Multiple range and multiple Ftestes. Biometrics, 11: 1-42.

EL-Ayek, M. Y. (1999). Influence of substituting concentrate feed mixture by Nigella sativa meal on: 1- Voluntary intake, digestibility, some rumen parameters and microbial protein yield with sheep. Egypt J. Nutr. and Feeds, 2: 279-296.

El-Hammady, H. Y. and A. A. A. Abdel-Kareem (2015). Reproductive performance of rabbit does producing low number of weaned kids treated with some dried herbal seeds. Egypt. Poult. Sci., 35(II): 609-625.

El-Khilany, F. M., S. G. Hammody, K. El-Bandr and S. Kazem (2009). Effect of adding Karkade to laying hen diets on the productive performance and egg quality measurements during summer season. Egyptian J. Nutr. and Feeds, 12(3) Special Issue: 771-779.

El-Kholy, K. H. S. Z. El-Damrawy and T. S. T. Seleem (2012). Rabbit productivity and reproductivity as affected by cinnamon (Cinnamomum zeylanicum). Egyptian Poultry Science, 32(6): 691-703.

Evans, F. J. and B. Pharm (1975). Herbs, A Concise Guide. (Cited by Abd El-Latif et al., 2002).

Ewuola, E. O., O. A. Sokunbi, T. K. Imam and C. U. Amadi (2011). Influence of dietary prebiotics and probiotics on blood profile of rabbits. Proc. 36th Ann. Conf. Nig. Soc. Anim. Produc.13th - $16^{\text {th }}$ March 2011 University of Abuja, Nigeria. Pp $171-173$

Gaafar, H. M. A., A. A. Ragab and K. F. A. El-Reidy (2014). Effect of diet supplemented with pumpkin (Cucurbita moschata) and black seed (Nigella sativa) oils on performance of rabbits: 2 - Productive and reproductive performance of does and their offspring. Rep. Opin., 6: 6068.

Gaya, I., O. Mohammad, A. Suleiman, M. Maje and A. Adekunle (2009).Toxicological and lactogenic studies on the seeds of Hibiscus sabdariffa L. (malvaceae) extract on serum prolactin levels of Albino Wistar Rats. Int. J. Endocrinology. 5(2): 540-2592.

Ghazalah, A. A. and A. M. Ali (2008). Rosemary leaves as a dietary supplement for growth in broiler chickens. Int. J. Poult. Sci., 7(3): 234-239.

Guo, F. C. (2003). Mushroom and herb polysaccharides as alternative for antimicrobial growth promoters on poultry. Ph.D. Thesis, Wageningen Institute of Animal Sciences, Dep. Anim. Nutr., Wageningen Univ., Wageningen, Netherlands.
Ibrahim, Sh. A. M. (2005). Effect of some medicinal plants as feed additives on growth and some metabolic changes in rabbits. Egyptian J. Nutr. and Feeds, 8: 207-219.

Ibrahim, Sh. A. M., A. T. El-Yamany and A. M. M. Zeid (2004). Dill and parsley as growth promoters in White New Zealand growing rabbits. Egypt. Poult. Sci., 24: 917-927.

Lukefahr, S. D. and P. R. Cheeke (1991). Rabbit project development strategies in subsistence farming systems. World Animal Review, 68: 60-70.

Mir, Z. P., S. N. Acharya, M. S. Zaman, W. G. Taylor, G. J. Mears, T. A. Mcallister and L. A. Goonewardene (1998). Comparison of alfalfa and fenugreek (Trigonella foenum graecum) silages supplemented with barley grain on performance of growing steers. Canadian J. Anim. Sci., 78: 343

Mohamed, M. M. (2009). Nuclear techniques to determine microbial protein synthesis and productive performance of Barki lambs fed rations containing some medicinal plants. M. Sc. Thesis, Fac. Agric., Cairo Univ., Egypt, 98pp.

Mukhtar, A. M. (2009). The effect of feeding rosella (Hibiscus sabdariffa) seed on broiler chick's performance. Research Journal of Animal and Veterinary Sciences, 2: 21-23.

NRC (1996). National Research Council. Nutrient Requirements of Rabbits. Washington, DC. USA.

Ocak, N., G. Erener, A. K. Burak, M. Sungu, A. Altop and A. Ozmen (2008).Performance of broilers fed diets supplemented with dry peppermint (Mentha piperita L.) or thyme (Thymus vulgaris L.) leaves as growth promoter source. Czech J. Anim. Sci., 53(4): 169-175.

Okasha, M. A., M. S. Abubakar and I. G. Bako (2008). Study of the effect of aqueous Hibiscus sabdariffa Linn seed extract on serum prolactin level of lactating female albino rats. European J. Sci. Res., 22(4): 575-583.

Omer, H. A. A., Y. A. A. EL-Nomeary, R. I. EL-Kady, Azza M. M. Badr, F. A. F. Ali, Sawsan M. Ahmed, H. M. H. EL-Allawy and Sh. A. M. Ibrahim (2013). Improving the utilization of rabbit diets containing vegetable oil by using fennel (Foeniculum vulgare) and oregano (Origanum vulgare L) as feed additives. Life Science Journal, 10(1): 2625-2636.

Osman Mona, H. M., Motawe H. F. Yakout and W. F. Ezz El-Arab (2010). Productive, physiological, immunological and economical effects of supplementing natural feed additives to broiler diets. Egypt. Poult. Sci., 30(1): 25-53.

Othman, A. A. (2005). Effect of some natural antioxidants on lambs performance. M.Sc. Thesis, Animal Production Department, Faculty of Agriculture, Cairo University.

Radwan, N. L. (2003). Effect of using some medicinal plants on performance and immunity of broiler chicks. Ph.D. Thesis, Animal Production 
Department. Faculty of Agriculture, Cairo University.

Rashwan, A. A. and I. F. M. Marai (2000). Mortality in young rabbits: A Review. World Rabbit Science, 8(3): 111-124.

Salem, F. A. and M. R. EL-Mahdy (2001). Effect of some medicinal plants as feed additives on nutrients digestibility, rumen fermentation, blood and carcass characteristics of sheep.2nd Int. Conf. Anim. Prod. Health in Semi-Arid Area, 161-175.

SAS (2004). SAS/STAT User's Guide.SAS Institute Inc., Cary, N.C.

Seleem, T. S. T., H. Ibrahim, A. M. Ali, A. M. AbdElmotaal and A. E. B, Zeidan (2007). Rabbit performance as affected by Origanum majorana in diets. 5th Int. Conf. Rabbit Production in Hot Climates, 8-10 Oct., Hurghada, Egypt, 495-508.

Shehata, E. I., Ferial H. Abd El-Rasoul, Faten F. Abou Ammou and A. M. Abdel-Gawad (2007). Effect of feeding the medicinal herb, chamomile flower, on some productive performance of Egyptian Zaraibi does and their new born kids. Egyptian J. Sheep and Goat Sci., 2(2): 111-120.

Tipu, M. A., M. S. Akhtar, M. I. Anjum and M. I Raja (2006). New dimension of medicinal plants as animal feed. Pakistan Vet. J., 26(3): 144-148.

\section{تأثير التظذية علي الحصالبان والبردقوش علي الأداء التناسلي لأمهات الأرانب تحت ظروف سيناء

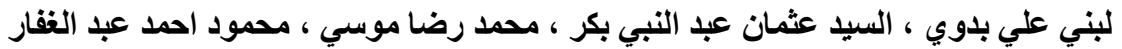

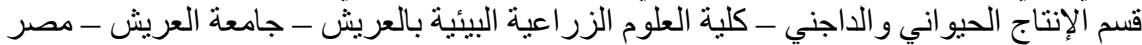

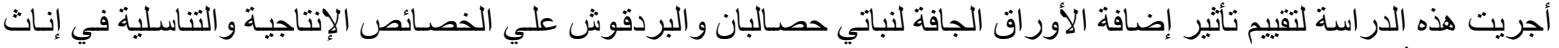

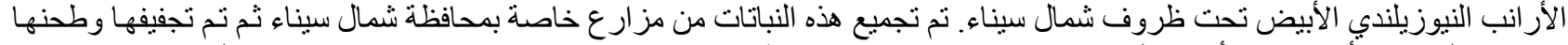

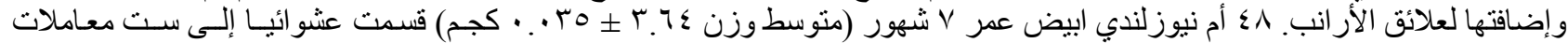

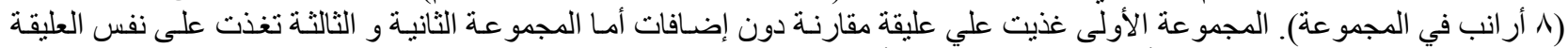

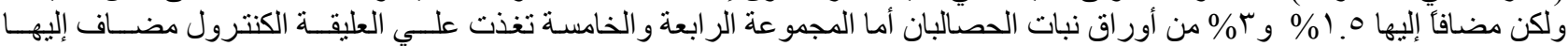

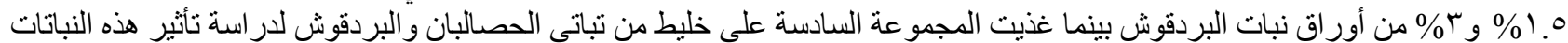

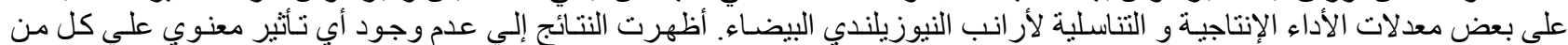

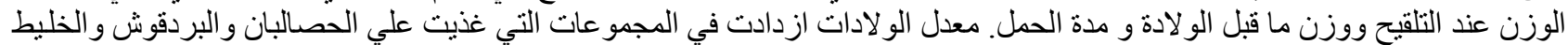

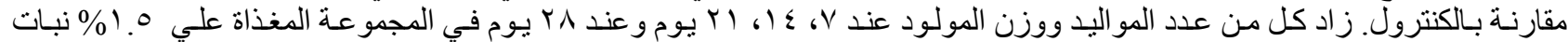

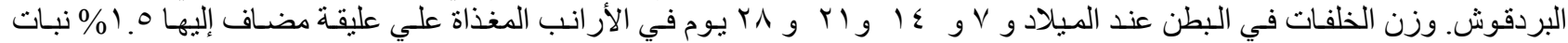

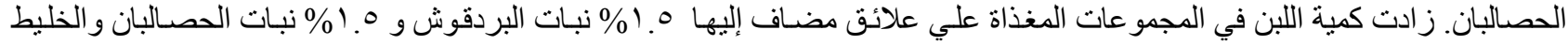

